\title{
A vertical structure of the edge-on galaxy, NGC 891
}

\author{
Toshihiro HANDA \&.Yoshiaki SOFUE \\ University of Tokyo, Institute of Astronomy
}

\author{
Satoru IKEUCHI \\ National Astronomical Observatory \\ Sumio ISHIZUKI \& Ryohei KAWABE \\ Nobeyama Radio Observatory
}

Most of observational researches on molecular gas in galaxies have focused on the twodimensional distributions in galactic disks so far. In order to investigate vertical structure of the galactic disk and the disk-halo interaction we need high-resolution observations of edge-on galaxies.

So we observed the nearest edge-on galaxy, NGC 891, using the Nobeyama Millimeter Array (NMA). Its distance is estimated to be $8.9 \mathrm{Mpc}$ using the Tully-Fisher relation and $\mathrm{H}$ band photometric data. The synthesized beamsize was $4.5^{\prime \prime} \times 4.4^{\prime \prime}$ and the field of view due to attenuation of the element antenna (Handa et al. 1992). The field center is 90 "-offset from the center of the galaxy along the major axis toward the northern side, where the $\mathrm{CO}$ intensity has a local maximum (Sofue et al. 1987). After the standard data reduction procedure in AIPS, including CLEAN, we obtained 10 channel-maps with a $19.5 \mathrm{~km} \mathrm{~s}^{-1}$ velocity width from $289.8 \mathrm{~km} \mathrm{~s}^{-1}$ to $465.2 \mathrm{~km} \mathrm{~s}^{-1}$ with respect to the local standard of rest.

In the integrated intensity map a narrow $\mathrm{CO}$ emission ridge is seen (Figure 1). It is an edge-on view of molecular gas disk of the galaxy. The confinement of the $\mathrm{CO}$ emission in the thin disk suggests that most of the molecular gas is belonging to the population-I objects like in our Galaxy. The apparent thickness of the ridge is about 7", which means that the deconvolved width is about 6" or $270 \mathrm{pc}$ (FWHM). The position-velocity diagram averaged along the major axis over the field of view shows no systematic velocity gradient along the minor axis (Figure 2). It means that the deconvolved thickness of the $\mathrm{CO}$ disk is intrinsic. This width is broader than that of our Galaxy by factor 2 . The geometrically thick CO disk of NGC 891 may be due to active star formation in the disk.

We found a prominent spur at $v_{\mathrm{LSR}}=407 \mathrm{~km} \mathrm{~s}^{-1}$. We call it as a "molecular spur" of NGC 891. The height of the spur is $520 \mathrm{pc}$ above the disk. Using the HI rotation curve of the galaxy, it is located at $5.5 \mathrm{kpc}$ from the galactic center. The mass of the spur is estimated to be $3 \times 10^{7} \mathrm{M}_{\odot}$, which is about a half mass of 30 Doradus molecular cloud complex.

We consider possible explanations about the origin of the molecular spur. The galaxygalaxy interaction is not, because NGC 891 has no companion and shows no warping in the outer HI disk. A falling cloud elongated by tidal force is not, because gravitation above a uniform flat disk does not generate tidal elongation. Therefore we think the spur was made by internal activity of the galaxy like some off-plane features in our Galaxy. The gravitational potential energy of the spur is estimated $3 \times 10^{52}$ erg using mass model of the galactic disk. The chimney model predicts a similar structure but predicted kinetic energy by superbubble is 
shortage about factor of 10 (Norman \& Ikeuchi 1989). Halo gas gathering due to the Parker instability is another possibility (Shibata \& Matsumoto 1991). If it is, then quick gas conversion from $\mathrm{HI}$ to $\mathrm{H}_{2}$ should be needed.

\section{References}

Handa,T., Sofue,Y., Ikeuchi,S., Kawabe,R., \& Ishizuki,S. 1992, PASJ, 44, L227

Norman,C.A., \& Ikeuchi,S. 1989, ApJ, 345, 372

Shibata,K., \& Matsumoto,R. 1991, Nature, 353, 633

Sofue,Y., Nakai,N., \& Handa,T. 1987, PASJ, 39, 47
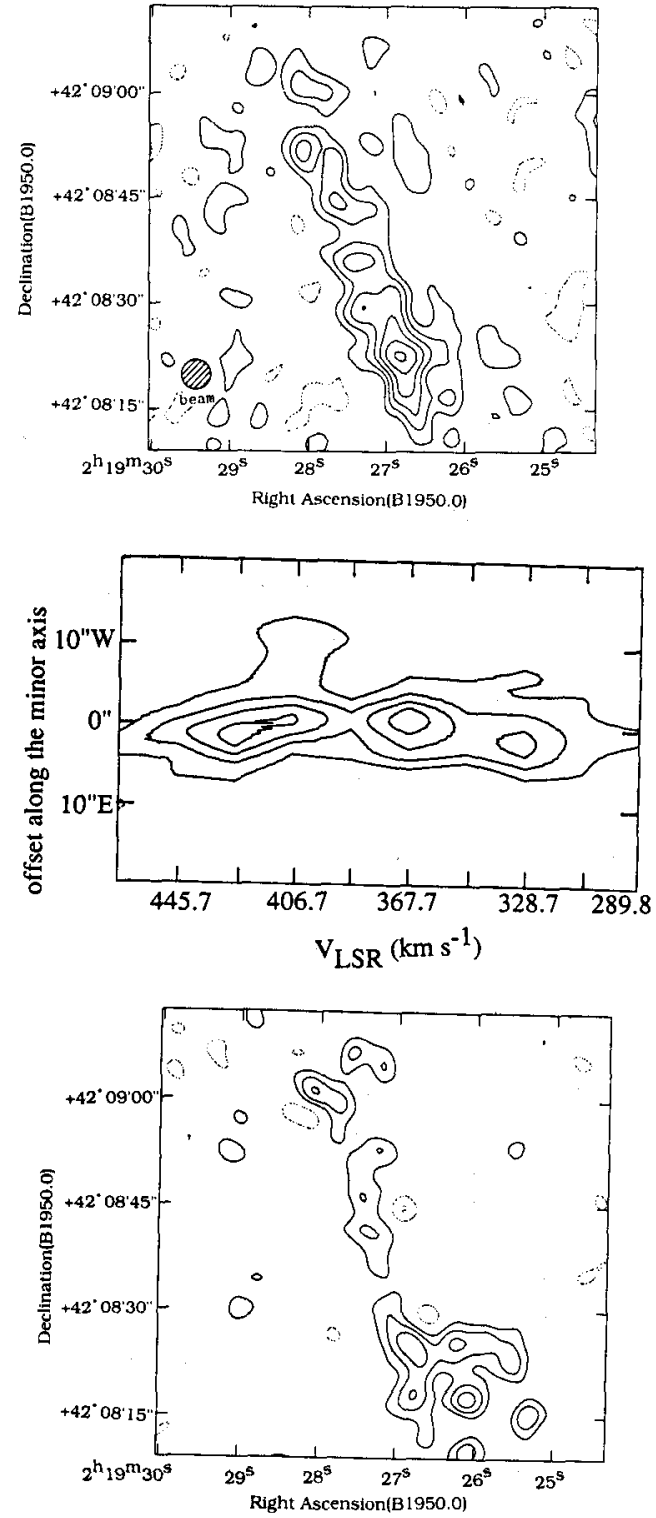

Figure 1: An integrated intensity map of NGC 891. The field center is 90" offset from the center of the galaxy.

Figure 2: A position-velocity map of NGC 891 along the minor axis averaged along the major axis. No velocity gradient which should be due to the inclined disk is seen. It means that the disk thickness is intrinsic.

Figure 3: A channel map at $v_{L S R}=407 \mathrm{~km} \mathrm{~s}^{-1}$. The molecular spur is seen around $\alpha=2^{h_{1}} 9^{m_{2}} 6^{s}$, $\delta=+42^{\circ} 8^{\prime} 25^{\prime \prime}$, which is $520 \mathrm{pc}$ above the galactic disk. 